\title{
CELL AND MOLECULAR BIOLOGY FOR NEUROSURGEONS (Everything you ever wanted to know about molecular biology but were afraid to download)
}

\section{Introduction by Topic Editors, Lawrence S. Chin, M.D., and Howard M. Eisenberg, M.D.}

We present in this issue of Neurosurgical Focus six review papers that outline the basic concepts of molecular biology and how they are being used to study neurosurgical diseases. We begin with an overview of basic molecular biology techniques including their rationale and methodology. To enhance reader comprehension, a unique feature has been added: a "pop-up" glossary of unfamiliar terms not described in the text. By placing a cursor over the underlined item, the reader will see a brief description appear on the screen without having to scroll to a glossary page. In the next review, Tang and Chiocca outline the rapidly expanding field of virus-mediated gene transfer. Central nervous system diseases will be one of the most important targets for gene therapy. Molecular techniques have changed our understanding of cerebral vasospasm. Thomas reviews the role of endothelin and nitric oxide in the pathogenesis of vasospasm from subarachnoid hemorrhage. For the past 10 years, cerebral tumors have been intensively studied using modern molecular biology techniques. Litofsky and Recht summarize the current understanding of the role of the $\mathbf{p 5 3}$ tumor suppressor gene in malignant gliomas. The role of growth factors in nervous system development and glioma growth is reviewed by Lazio and Chin. Finally, Harter, et al., review the detection and regulation of apoptosis. After finishing this series of reviews, the reader should have a broader understanding of the role of molecular biology techniques in the neurosciences. We encourage you to download these articles and to invite others to access this Web site. 